\title{
Brote de queratoconjuntivitis epidémica en un Servicio de Oftalmología
}

\author{
Patricio Nercelles M., Luisa Peirano N., Rosa Herrera O, Pamela Rivero B. y Leonor Márquez P.
}

\begin{abstract}
Hospital Carlos Van Buren, Valparaíso, Chile.

Unidad de Calidad y Seguridad del Paciente (PNM, LPN, RHO, PRB, LMP).

Universidad de Valparaíso,

Chile.

Facultad de Medicina

Departamento de Microbiología (PNM).
\end{abstract}

Recibido: 31 de mayo de 2010 Aceptado: 5 de septiembre de 2010

Correspondencia a: Patricio Nercelles Muñoz patricio.nercelles@uv.cl

\section{A nosocomial outbreak of epidemic keratoconjunctivitis}

We describe a nosocomial outbreak of keratoconjunctivitis at the Ophthalmology Clinic in Hospital Carlos Van Buren, that affected 150 patients, during August and September of 2006. This outbreak was concomitant with a community outbreak produced by the same agent in the city of Valparaiso that affected 681 other patients. Although adenovirus was not isolated in the nosocomial cases, positive cultures were obtained from community cases, so the diagnosis was based on clinical and epidemiological criteria. The infection rate within the Clinic patients was $5.2 \%$ considering the population that attended the Clinic in this period. The evaluation of patient care practices showed that common risk factors among affected cases were measurement of ocular tension with a tonometer in the Ophthalmology Clinic (without disinfection of the tonometer between patients), contamination of work surfaces (equipment, furniture), and poor compliance of hand hygiene. Control measures adopted were cleaning, disinfection of tonometer, equipment and work surfaces, and reinforcement of hand hygiene measures. With these measures, it was possible to control the nosocomial outbreak, despite the continued outpatient care of community-acquired cases.

Key words: Epidemic keratoconjunctivitis, adenovirus, contact precautions.

Palabras clave: Queratoconjuntivitis, adenovirus, brote nosocomial, precauciones de contacto.

\section{Introducción}

L a queratoconjuntivitis epidémica (QCE) es una infección aguda que se presenta en forma esporádica o en brotes a partir de una fuente común, en cualquier época del año. En la comunidad se ha asociado a agua de piscinas con baja concentración de cloro y en los casos de brotes nosocomiales se han presentado en unidades de neonatología, pediatría y oftalmología, asociados a malas prácticas de atención. El cuadro clínico se manifiesta inicialmente como una conjuntivitis aguda con epífora, ojo rojo, malestar y fotofobia, bilateral en $\sim 60 \%$ de los casos. Al examen se observa edema palpebral con secreción acuosa, quemosis y reacción folicular conjuntival; en casos graves también hemorragias subconjuntivales, pseudomembranas y linfadenopatía. La queratitis aparece días después y se manifiesta con sensación de arenilla, disminución de la agudeza visual, fotofobia, epífora, blefarospasmo y, en un tercio de los casos, aparecen infiltrados subepiteliales, una de las complicaciones más graves, por persistir con estas molestias durante meses y años ${ }^{1}$.

La infección se transmite por contacto persona a persona $y$, en casos nosocomiales en general, a través de las secreciones oculares de los pacientes y de artículos de la atención contaminados tales como tonómetros, lámpara de hendidura, blefarostatos, mobiliario y manos del personal. El período de incubación es de 4 a 10 días y el período de transmisibilidad dura hasta 14 días desde el inicio de la enfermedad ${ }^{1,2}$.

La mayoría de los brotes epidémicos publicados presenta una fuente de infección común como la consulta oftalmológica y/o algún instrumento relacionado a la atención. Las manos del personal de salud juegan un rol importante en la propagación de la enfermedad ${ }^{2}$.

Adenovirus (ADV) es un virus de tamaño mediano (90 a $100 \mathrm{~nm}$ ), sin envoltura, de 16 lados, con ADN de doble hebra. Existen 49 tipos inmunológicamente distintos (en 6 subgéneros: nombrados del A al F) que pueden causar una enfermedad en seres humanos. Los ADV generalmente son estables contra agentes químicos o físicos y pueden sobrevivir varios días en las superficies ${ }^{3,4}$. La QCE está asociada con los serotipos 8, 19 y 37 .

El diagnóstico se puede realizar por detección del antígeno, pruebas de reacción de polimerasa en cadena (RPC), aislamiento en cultivo celular y serología. La tipificación se realiza por pruebas de inhibición de la hemaglutinación y/o neutralización con antisueros específicos ${ }^{4}$.

Objetivo. Describir la presentación del brote de QCE en el tiempo, los factores de riesgo detectados y el impacto de las medidas de control. 


\section{Pacientes y Método}

Estudio descriptivo y retrospectivo, basado en la revisión de fichas clínicas y formularios de atención de urgencia en la Policlínica de Oftalmología del Hospital Carlos Van Buren, Valparaíso, durante los meses de agosto y septiembre de 2006. Se definió como caso del brote a todo paciente con cuadro clínico de queratoconjuntivitis que había sido atendido previamente en la Policlínica de Oftalmología por otra patología. La información de los potenciales casos se obtuvo del listado diario de pacientes atendidos en esta policlínica considerándose como población expuesta al total de pacientes atendidos en la misma policlínica y en el mismo período. Al mismo tiempo se revisó en todos los pacientes con diagnóstico de QCE si tenían consultas previas en el mismo servicio, para descartar los casos de la comunidad. El estudio virológico (cultivo celular e identificación con anticuerpos monoclonales), se realizó en el Laboratorio de Virología de la Facultad de Medicina de la Universidad de Valparaíso. La supervisión de las prácticas de atención directa en la Policlínica de Oftalmología se realizó mediante pautas de cotejo. Aparte del género y edad no se evaluaron otros aspectos de los pacientes, ya que la mayoría de ellos fueron atendidos como urgencias por lo que no quedaron registros en la ficha clínica; tampoco se hizo seguimiento en busca de complicaciones posteriores.

\section{Resultados}

A mediados de agosto del 2006 se hospitalizaron tres pacientes para cirugías oftalmológicas electivas, diagnosticándose al ingreso en todos ellos queratoconjuntivitis. Estos pacientes habían sido atendidos previamente en la Policlínica de Oftalmología, que en esa misma fecha presentaba un alto número de consultas por QCE en personas provenientes de la comunidad. Por la sospecha de brote se realizó revisión de las consultas en las semanas previas, comprobándose la existencia de casos nosocomiales una semana antes de los casos índices. El número total de casos en los meses de agosto y septiembre llegó a 150 de un total de 2.886 pacientes atendidos, lo que representó una tasa de 5,2\%. La curva epidémica muestra el inicio del brote a principios de agosto con un ascenso sostenido y que alcanzó el máximo de casos al final del mes, para seguir con un descenso brusco coincidente con las medidas ambientales de prevención, detectándose el último caso la tercera semana de septiembre (Figura 1).

Por otro lado, el brote en la comunidad se inició en enero del mismo año y tuvo una presentación bimodal con máximos en los meses de febrero y agosto, alcanzando un total de 681 pacientes a fines de diciembre (Figura 2) (Fuente: Dpto. Epidemiología. SS Valparaíso, comunicación personal).

\section{Supervisión de prácticas de atención}

En la Policlínica de Oftalmología se detectó falta de desinfección de los tonómetros, los que eran limpiados previamente con éter entre paciente y paciente y que por haberse terminado sólo se limpiaban con algodón. Por la ausencia de registros no se pudo determinar qué pacientes habían estado expuestos a este procedimiento (se realizaban más de 50 mediciones diarias). Por las características de supervivencia del virus en superficies se consideró que todos los equipos de oftalmología estaban potencialmente contaminados (lámparas de hendidura, tonómetros, campimetría), los que por lo demás no contaban con ningún protocolo de limpieza y desinfección periódica; además se comprobó falta de higienización de manos durante la atención, tanto de los profesionales como del resto del personal.

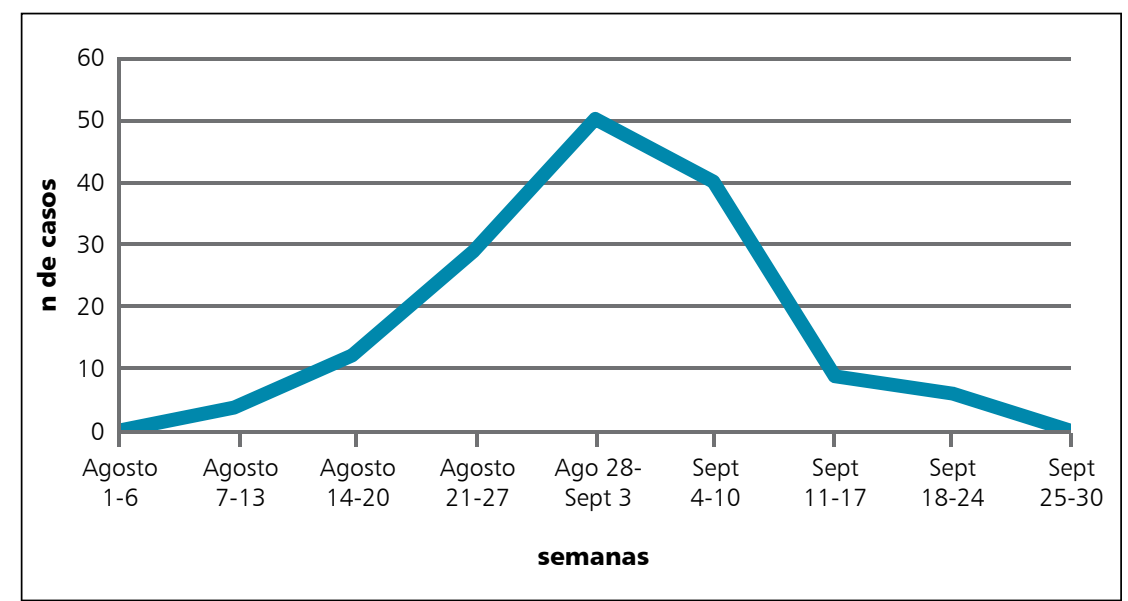

Figura 1. Curva epidémica de casos de queratoconjuntivitis. Servicio Oftalmología. Hospital Carlos Van Buren año 2006.

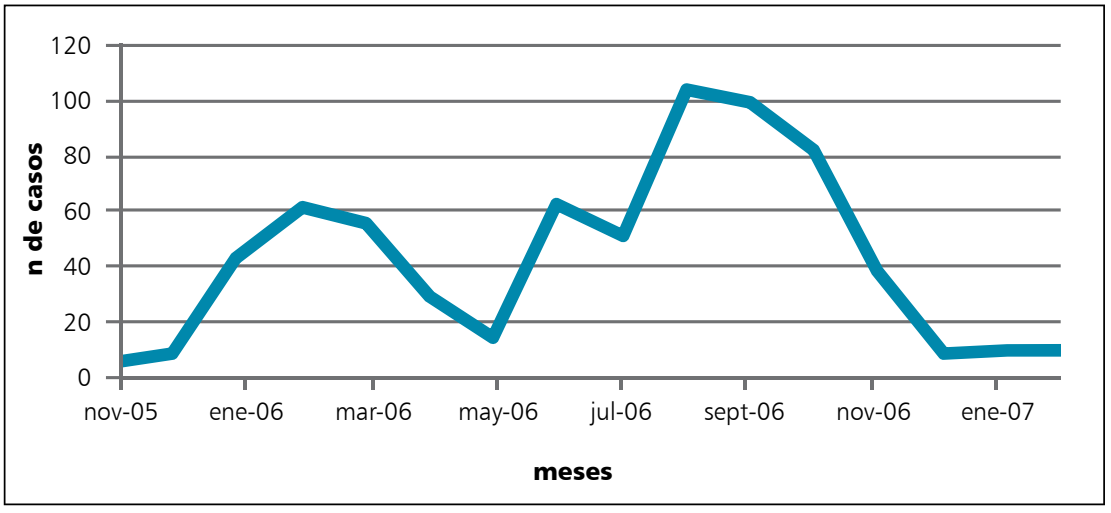

Figura 2. Curva epidémica de casos de queratoconjuntivitis epidémica. Ciudad de Valparaíso año 2006. 
La gran mayoría de los casos del brote intrahospitalario correspondió a adultos, con predominio de mujeres $(60,5 \%)$, el tiempo promedio entre la primera consulta y la consulta por QCE fue de 12,9 días con un rango entre 5 y 27 días. Sólo se logró enviar a cultivos virales diez muestras adecuadas y se documentó la existencia de ADV en tres de seis pacientes con QCE de la comunidad (sin estudio de serotipo), no se obteniéndose cultivos positivos en las cuatro muestras de casos del brote nosocomial.

\section{Medidas de control del brote nosocomial}

En cuanto se sospechó del brote se tomaron medidas dirigidas a controlar las fuentes de infección, considerando el importante reservorio diario de más de 30 pacientes con QCE proveniente de la comunidad. Las medidas consistieron en la implementación de un protocolo de limpieza y desinfección con alcohol en todos los equipos, higienización de manos entre pacientes y antes de realizar algún procedimiento. Con estas medidas no se logró el control del brote, por lo que a la tercera semana se instauraron medidas adicionales de limpieza y desinfección con clorados en las superficies de muebles, paredes, puertas de las salas de atención, de espera y baños de la policlínica de la especialidad. Al mismo tiempo se realizaron charlas a todo el personal para dar a conocer los reservorios y vías de transmisión como asimismo las medidas de prevención. Además se dio a conocer los resultados de la supervisión del cumplimiento de las prácticas. Con estas medidas adicionales se logró controlar el brote, no presentándose nuevos casos desde la última semana de septiembre.

\section{Discusión}

Se describe uno de los brotes con el mayor número de casos notificados en el país, que afectó a 150 pacientes con QCE por ADV en el Servicio de Oftalmología durante los meses de agosto y septiembre de 2006, en el contexto de un brote comunitario en la ciudad de Valparaíso, también con gran número de casos. En la investigación del brote se detectó una serie de falencias que permitieron la aparición y mantención del brote. Al establecerse las medidas de control (precauciones de contacto), el brote pudo ser controlado más rápidamente si se compara con otros brotes publicados. Una de las particularidades de este brote es que no hubo personal de salud afectado y que no se diseminó más allá del Servicio de Oftalmología.

Este agente es de alta contagiosidad y su permanencia en las superficies favorece su diseminación, por lo que el manejo de las superficies con limpieza y desinfectantes de bajo nivel como clorados o amonios cuaternarios es muy importante en las medidas de control. Asimismo se ha recomendado como efectiva la desinfección de los equipos como tonómetros con alcohol isopropílico ${ }^{5}$.

El año 2005 se notificó un brote de QCE en la Unidad de Neonatología en nuestro hospital, que afectó a cuatro neonatos y siete funcionarios del hospital (una matrona y seis técnicos paramédicos). Este brote se relacionó con un blefaróstato procesado entre paciente y paciente en la misma Unidad y a fallas en el lavado de manos. Este brote permitió cambiar el procesamiento del blefaróstato, que actualmente se realiza en el Servicio de Esterilización.

Las características y factores de riesgo del brote nosocomial presentado en esta comunicación es concordante con otros brotes producidos por este agente etiológico. La revisión de la literatura médica permitió identificar 27 brotes de QCE publicados entre los años 1984 y $2009^{1,2,6-30}$, la mayoría correspondió a los serotipos 8, 37 y 7 y afectaron a adultos $(65 \%)$ y neonatos $(26 \%)$, de Servicios de Oftalmología (52\%) y Neonatología (26\%). Por otra parte, también en su gran mayoría, el mecanismo de transmisión fue a través de las manos del personal, equipos y superficies contaminadas. La mayoría de los brotes afectó a pacientes y personal de salud (59\%). En adultos el cuadro clínico estuvo más limitado a la QCE y en niños además a cuadros respiratorios altos y bajos. El promedio de pacientes afectados por brote fue de 41 (3-200) y en el personal fue de 10,7 (1-43). A su vez, la duración promedio de los brotes fue de 3,8 (1-12) meses. Las medidas de control se focalizaron en la higiene de manos, desinfección de equipos y superficies, uso de guantes, delantal y aislamiento en cohorte (Tabla 1).

En Chile, de acuerdo a la información del Ministerio de Salud, se han notificado 43 brotes nosocomiales producidos por ADV entre los años 1986 y 2006, que afectaron a 653 pacientes, de los cuales fallecieron 59, con una letalidad de $9 \%$. La gran mayoría de los brotes se produjeron en servicios pediátricos y asociados a cuadros respiratorios bajos y altos. Algunos de estos brotes estuvieron relacionados con epidemias de la comunidad al igual que el presente y también se identificó como causa probable la transgresión de prácticas, en especial higienización de $\operatorname{manos}^{31}$. Recientemente se publicó un brote de QCE en el país, de similares características y en el mismo período de tiempo de este reporte ${ }^{32}$.

La información y características del presente brote permite visualizar la presencia de problemas específicos de infecciones intrahospitalarias (IIH) que no son abordados ni identificados sistemáticamente en los hospitales fundamentalmente debido a la percepción que se trata de servicios de "bajo riesgo". En estos servicios, por lo general no existen sistemas de vigilancia de IIH y no son objeto de programas de capacitación ni supervisión. Lo anterior hace que probablemente la transgresión en medidas básicas de prevención tales como higienización de manos y procesamiento de artículos entre pacientes sea mayor que en otros servicios, de tal manera que apareciendo un reservorio sea muy fácil la aparición de situaciones como las ya descritas. 
Este brote demuestra además que el conocimiento de la vía de transmisión y de las medidas de control permite enfrentar esta emergencia sin recurrir a cierre de los servicios, lo que sin duda produciría un daño mayor a la población que el mismo brote.

Por las características de este estudio no es posible establecer los factores de riesgo de este brote pero la detección de fallas en las prácticas de atención mediante la supervisión, y el control del brote cuando se aplicaron correctamente, permite inferir que jugaron un rol importante la falta de higiene de manos, fallas en la desinfección de equipos y superficies.

Sería recomendable que los programas locales de prevención de infecciones incorporen también las áreas clínicas de menor riesgo, considerando factores de riesgo específicos.

\section{Resumen}

Se describe un brote nosocomial de queratoconjuntivitis epidémica en la Policlínica de Oftalmología del Hospital Carlos Van Buren, que afectó a 150 pacientes, durante los meses de agosto y septiembre de 2006. Este brote se presentó en el contexto de un brote en la ciudad de Valparaíso que afectó a otros 681 pacientes. Aun cuando no se logró aislar adenovirus (ADV) en los casos nosocomiales, se aisló ADV en pacientes del brote comunitario, por lo que el diagnóstico de los casos nosocomiales se basó en criterios clínicos y epidemiológicos. La tasa de ataque del brote nosocomial fue de 5,2\% considerando la población consultante del período. La evaluación de prácticas de atención directa mostró que los factores de riesgo de los casos serían la medición de la tensión ocular mediante tonómetro en la Policlínica de Oftalmología (sin desinfección del tonómetro entre paciente y paciente), contaminación de superficies de trabajo (equipos, mobiliario) y bajo cumplimiento de la higienización de manos. Las medidas de control fueron limpieza, desinfección del tonómetro, equipos y superficies de trabajo, junto a refor-
Tabla 1. Características de 27 brotes publicados de queratoconjuntivitis por adenovirus

\begin{tabular}{|c|c|c|}
\hline Grupos de edades & $\begin{array}{l}\text { Adultos } \\
\text { Neonatos } \\
\text { Adultos/niños } \\
\text { Niños }\end{array}$ & $\begin{array}{l}17(65 \%) \\
6(23 \%) \\
2 \\
1\end{array}$ \\
\hline Servicios clínicos & $\begin{array}{l}\text { Oftalmología } \\
\text { Neonatología } \\
\text { Pediatría } \\
\text { Otros }\end{array}$ & $\begin{array}{l}14(52 \%) \\
7(26 \%) \\
2 \\
4\end{array}$ \\
\hline Población afectada & $\begin{array}{l}\text { Personal y pacientes } \\
\text { Sólo pacientes } \\
\text { Sólo personal }\end{array}$ & $\begin{array}{l}16(59 \%) \\
8(30 \%) \\
3\end{array}$ \\
\hline $\begin{array}{l}N^{\circ} \text { casos en paciente } \\
N^{\circ} \text { casos en personal de salud }\end{array}$ & $\begin{array}{l}\text { Rango } 3 \text { a } 200 \\
\text { Rango } 1 \text { a } 43\end{array}$ & $\begin{array}{l}\text { Promedio } 41 \\
\text { Promedio } 10,7\end{array}$ \\
\hline Localización de la infección & $\begin{array}{l}\text { Conjuntivitis } \\
\text { Respiratoria superior } \\
\text { Respiratoria inferior }\end{array}$ & $\begin{array}{r}27 \\
11 \\
4\end{array}$ \\
\hline Transmisión & $\begin{array}{l}\text { Contacto } \\
\text { Equipos médicos } \\
\text { Manos } \\
\text { No mencionado }\end{array}$ & $\begin{array}{r}12 \\
5 \\
4 \\
6\end{array}$ \\
\hline Serotipos & $\begin{array}{l}8 \\
37 \\
7 \\
\text { Otros }\end{array}$ & $\begin{array}{l}14(67 \%) \\
3 \\
2 \\
2\end{array}$ \\
\hline Duración del brote (meses) & Rango 1 a 12 & Promedio 3,8 \\
\hline Medidas de control* & $\begin{array}{l}\text { Refuerzo de lavado de manos } \\
\text { Desinfección de equipos médicos } \\
\text { Desinfección de superficies } \\
\text { Aislamiento en cohorte } \\
\text { Barreras (guantes, delantal, lentes) } \\
\text { Restricción laboral } \\
\text { Cierre de servicios }\end{array}$ & $\begin{array}{r}14 \\
11 \\
10 \\
10 \\
8 \\
3 \\
2\end{array}$ \\
\hline
\end{tabular}

zamiento de lavado de manos. Con estas medidas se logró controlar el brote intrahospitalario a pesar de mantenerse las consultas provenientes del brote en la comunidad.

\section{Referencias}

1.- Asencio-Durán M, Romero-Martin R, García-Martínez J R, Peralta-Calvo J M, Pérez-Blanco V, García-Caballero J, et al. Nosocomial outbreak of epidemic keratoconjunctivitis in a neonatal intensive care unit. Arch Soc Esp Oftalmol 2007; 82: 73-9.

2.- Salcedo M A, Goldaracena B, Ardanaz M E, Mazon A, Moreno C, Salvo S. Nosocomial and community outbreak of epidemic keratoconjunctivitis in Navarra in 1996. Rev Esp Salud Publica 1997; 71: 383-90.
3.- Gordon Y J, Gordon R Y, Romanowski E, Araullo-Cruz T P. Prolonged recovery of desiccated adenoviral serotypes 5,8 , and 19 from plastic and metal surfaces in vitro. Ophthalmology 1993; 100: 1835-9.

4.- Adenoviruses.www.cdc.gov/ncidod/dvrd/revb/ respiratory/eadfeat-spanish.htm. (Accedido el 3 de mayo 2010).

5.- Rutala W A, Peacock J E, Gergen M F, Sobsey M D, Weber D J. Efficacy of hospital germicides against adenovirus 8 , a common cause of epidemic keratoconjunctivitis in health care facilities. Antimicrob Agents Chemother.
2006; 50: 1419-24

6.- Richmond S, Burman R, Crosdale E, Cropper L, Longson D, Enoch B E, et al. A large outbreak of keratoconjunctivitis due to adenovirus type 8 . J Hyg (Lond) 1984; 93: 285-91.

7.- Porter J D, Teter M, Traister V, Pizzutti W, Parkin W E, Farrell J. Outbreak of adenoviral infections in a long-term paediatric facility, New Jersey, 1986/87. J Hosp Infect 1991; 18: 201-10.

8.- Finn A, Anday E, Talbot G H. An epidemic of adenovirus 7a infection in a neonatal nursery: course, morbidity, and management. Infect Control Hosp Epidemiol 1988; 9: 398-404. 
9.- Piedra P A, Kasel JA, Norton H J, García-Prats J A, Rayford Y, Estes M K, et al. Description of an adenovirus type 8 outbreak in hospitalized neonates born prematurely. Pediatr Infect Dis J 1992; 11: 460-5.

10.- Ankers H E, Klapper P E, Cleator G M, Bailey A S, Tullo A B. The role of a rapid diagnostic test (adenovirus immune dot-blot) in the control of an outbreak of adenovirus type 8 keratoconjunctivitis. Eye 1993; 7: 15-7.

11.- Jernigan J A, Lowry B S, Hayden F G, Kyger S A, Conway B P, Gröschel D H, et al. Adenovirus type 8 epidemic keratoconjunctivitis in an eye clinic: risk factors and control. J Infect Dis 1993; 167: 1307-13.

12.- Tabery H M. Two outbreaks of adenovirus type 8 keratoconjunctivitis with different outcome. Acta Ophthalmol 1995; 73: 358-60.

13.- Azar M J, Dhaliwal D K, Bower K S, Kowalski R P, Gordon Y J. Possible consequences of shaking hands with your patients with epidemic keratoconjunctivitis. Am J Ophthalmol 1996; 121: 711-2.

14.- Chaberny I F, Schnitzler P, Geiss H K, Wendt C. An outbreak of epidemic keratoconjunctivitis in a pediatric unit due to adenovirus type 8 . Infect Control Hosp Epidemiol 2003; 24: 514-9.

15.- Imai Y, Kameya S, Ohkoshi M, Yamaki K, Sakuragi S. Identification of the hexon region of an adenovirus involved in a new outbreak of keratoconjunctivitis. J Clin Microbiol 2001; 39: 2975-7.

16.- Piednoir E, Bureau-Chalot F, Merle C, Gotzamanis A, Wuibout J, Bajolet O. Direct costs associated with a nosocomial outbreak of adenoviral conjunctivitis infection in a longterm care institution. Am J Infect Control 2002; 30: 407-10.

17.- Percivalle E, Sarasini A, Torsellini M, Bruschi L, Antoniazzi E, Grazia Revello M, et al. A comparison of methods for detecting adenovirus type 8 keratoconjunctivitis during a nosocomial outbreak in a Neonatal Intensive Care Unit. J Clin Virol 2003; 28: 257-64.

18.- Sendra-Gutiérrez J M, Martin-Ríos D, Casas I, Saez P, Tovar A, Moreno C. An outbreak of adenovirus type 8 keratoconjunctivitis in a nursing home in Madrid. Euro Surveill 2004; 9: 27-30.

19.- James L, Vernon M O, Jones R C, Steward A, Lu X, Zollar L M, et al. Outbreak of human adenovirus type 3 infection in a pediatric longterm care facility-Illinois, 2005. Clin Infect Dis 2007; 45: 416-20.

20.- Kaneko H, Maruko I, Iidia T, Ohguchi T. The possibility of human adenovirus detection from the conjunctiva in asymptomatic cases during nosocomial outbreak. Cornea 2008; 27: 527-30.

21.- Melendez C P, Florentino M M, Martínez IL, López H M. Outbreak of epidemic keratoconjunctivitis caused by adenovirus in medical residents. Mol Vis 2009; 15: 557-62.

22.- Hamada N, Gotoh K, Hara K, Iwahashi J, Imamura Y, Nakamura S, et al. Nosocomial outbreak of epidemic keratoconjunctivitis accompanying environmental contamination with adenoviruses. J Hosp Infect 2008; 68: 262-8.

23.- Yun H C, Prakash V. Transmission of adenovirus serotype 14 in the health care setting. Clin Infect Dis 2008; 46: 1935-6.

24.- Engelmann I, Madisch I, Pommer H, Heim A. An outbreak of epidemic keratoconjunctivitis caused by a new intermediate adenovirus $22 / \mathrm{H} 8$ identified by molecular typing. Clin Infect Dis 2006; 43: 64-6.

25.- Birembaum E, Linder N, Varsano N, Azar R, Kuint J, Spierer A, et al. Adenovirus type 8 conjunctivitis outbreak in a neonatal intensive care unit. Arch Dis Child 1993; 68: 610-11.

26.- Curtis S, Wilkinson G W, Westmoreland D. An outbreak of epidemic keratoconjunctivitis caused by adenovirus type 37. J Med Microbiol 1998; 47: 91-4.

27.- Montessori V, Scharf S, Holland Werker D H, Roberts F J, Bryce E. Epidemic keratoconjunctivitis outbreak at a tertiary referral eye care clinic. Am J Infect Control 1998; 26: 399-405.

28.- Faden H, Wynn R J, Campana L, Ryan R M. Outbreak of adenovirus type 30 in a neonatal intensive care unit. J Pediatr 2005; 146: 523-7.

29.- Cheung D, Bremner J, Chan JT. Epidemic kerato-conjunctivitis-do outbreaks have to be epidemic? Eye 2003; 17: 356-63.

30.- Klapper P E, Cleator G M. Adenovirus crossinfection: a continuing problem. J Hosp Infect 1995; 30: 262-7.

31.- Brotes de Infecciones Intrahospitalarias por adenovirus en Chile. Ministerio de Salud (comunicación personal de Pola Brenner).

32.- Romero P, Vogel M, Solanes F, Luchsinger V. Queratoconjuntivitis por adenovirus generadas a partir de una consulta oftalmológica. Rev Chil Infect 2010; 27 (2): 148-52. 